\title{
Mesenchymal Stem-Cells Patches Improve Burn Wound Reepithelization in a Porcine Wound Model: A Prospective, Comparative Study
}

\section{González Martínez PC, Baley Spindel I, Villasenor Villalpando E*, Garzon Muvdi J, Chávez Osorio D and Márquez Espriella C \\ Hospital Central Sur de Alta Especialidad, Petróleos Mexicanos, Mexico}

*Corresponding author: Eduardo Villaseñor-Villalpando, Hospital Central Sur de Alta Especialidad, Petróleos Mexicanos, Mexico City, Tel: +52-155-3966-5609; Email: dr.eduardovillasenor@gmail.com

\section{Conceptual Paper}

Volume 2 Issue 3

Received Date: October 24, 2018

Published Date: November 26, 2018

DOI: $10.23880 /$ ijtps-16000125

\section{Abstract}

Background: Delayed burn wound healing leads to increased morbidity, painful and unsightly scars. It is attributed to prolonged systemic and wound inflammation, vascular damage and hypoxia. Among current therapies, semi-occlusive dressings provide a moist environment, and cultured keratinocytes moderately hasten epithelization; however neither can control the pro-inflammatory response. Stem cells, in particular mesenchymal, accelerate the healing process. They promote local cell proliferation, tissue regeneration and angiogenesis. Their immuno-modulatory and anti-inflammatory properties allow for transplantation without histocompatibility matching. There are no pre-fabricated stem cell patches commercially available. We experimented with a tissue-engineered patch that delivers allogenic mesenchymal stem-cellsderived from the human endometrium - nested on a collagen scaffold; we assessed its effects on reeptithelization rates on burn wounds compared to semi-occlusive dressings and keratinocyte patches.

Methods: In a swine model burn wounds were made with a hot plate. Each was covered with either a semi-occlusive dressing, a cultured keratinocyte patch, or stem-cell patch. A grading scale was developed for histologic findings for stratum corneum, epidermis and dermis.

Results: There was no significant difference between semi-occlusive and keratinocyte patches. There was significant difference between stem-cell patches and semi-occlusive patches on all layers. Stem-cell patches were superior to keratinocytes only on the dermal layer.

Conclusions: Endometrium derived mesenchymal stem-cells are abundant and easy to harvest for prefabrication of live cellular dressings. They promote a faster and more organized wound healing, even in cross-species grafting. 


\section{International Journal of Transplantation \& Plastic Surgery}

Keywords: Burn Wound; Reeptithelization; Grafts; Mesenchymal stem-cells; Cross-species grafting

Abbreviations: ESC: Endometrial Stem Cells; MSCs: Mesenchymal Stem Cells.

\section{Introduction}

Delayed epithelization of burns and chronic wounds leads to higher risk of infection, chronic pain, increased number of surgical procedures, and prolonged hospital stays. It also results in hypertrophic and painful scars, and an overall worse cosmetic result. Prolonged epithelization periods are attributable to inflammatory conditions in the wound. Immediately after injury, the hypoxic state of the wound attracts circulating inflammatory cells; in order for these inflammatory phase to resolve, revascularization and cell proliferation are necessary [1]. However, due to vascular damage on burnt tissues and chronic wounds, this process takes more than usual.

Different therapies attempt to speed-up the wound healing process and control local inflammatory conditions. Among them, semi-occlusive dressings provide a moist environment for faster re-epithelization, however this effect is no different than traditional gauze dressings. Ointments and topical immuno-modulators are useful adjuvants.

Autologous or allogenic cell grafts have been proposed as a method to accelerate the healing process. Prefabricated cell cultures, mostly keratinocytes, are available either as suspensions or sheets to be applied over the wounds. Although they do provide faster recovery times, they lack the ability to inhibit the inflammatory process, which leads to high rates of graft loss and has little impact on the overall inflammatory state of the patient. They have the advantage of being readily available, but they are costly and difficult to store.

Stem cells are beneficial in accelerating the healing process of burns and chronic wounds [2]. In particular, mesenchymal stem-cells (MSCs) are the most promising because of their ability to differentiate into ectodermal, mesodermal or endodermal cells [3]. Furthermore, while wound fibroblasts have a pro-inflammatory cytokine profile, MSCs tends to secrete cytokines that promote local cell proliferation, tissue regeneration and angiogenesis. The microenvironment created by MSCs promotes keratinocyte and endothelial cell proliferation in vitro, and wounds treated with MSCs grow more skin appendages than fibroblasts alone [4].

There are over 400 FDA-approved clinical trials involving mesenchymal stem-cell grafting (either adult or embryonic, autologous or allogenic). MSC express HLA class I surface molecules, and even though they do express HLA class II molecules intracellularly, less than $10 \%$ express them on the surface after stimulation. Furthermore, MSC have strong immuno-modulatory, immuno-tolerant and anti-inflammatory properties, effectively inhibiting alloreactive lymphocyte reactions. All of these characteristics allow for successful transplantation with minimal incompatibility issues between individuals [5].

Until today there is no bio-technological product that effectively delivers live stem cells other than custom, prefabricated autologous grafts made from stem-cells previously harvested from the patient. This in turn limits production and availability. The optimal source and method for stem cell therapy in burns and chronic wounds has yet to be determined.

We experimented with a novel tissue-engineered patch that delivers allogenic mesenchymal stem-cells derived from the human endometrium nested a collagen scaffold; we assessed its effects on reeptithelization on burn wounds, and compared it with traditional semiocclusive dressings and cultured keratinocyte patches.

\section{Materials and Methods}

\section{Animals}

Approval from Hospital Central Sur de Alta Especialidad de Petróleos Mexicanos' Animal Care and Experimental Surgery Department was obtained for the protocol. Ten Yorkshire-Landrace pigs (Bioinvert, Mexico City.) weighing 10 to $16 \mathrm{~kg}$ were used for this study.

We chose a swine model for the experiment because pig and human skin are genetically compatible up to a $78 \%$, furthermore, many commercially available products for temporary wound coverage are made from pig skin. The skin characteristics and hair pattern of the pigs is also similar to that of humans: not too thick and hairy like dogs, nor to thin like rabbits and rats. 


\section{International Journal of Transplantation \& Plastic Surgery}

\section{Skin burns}

Pigs were anesthetized with Tiletamine/Zolazepam (Zoletil®, Virbac, Mex.), 3.5 mg IM; Xilazine (Procin ${ }^{\circledR}$ equus, Pisa, Mexico) $1.32 \mathrm{mg}$, IM; Tramadol (Tradol®, Grüenthal, Mexico) $50 \mathrm{mg}$ IM. IV penicillin prophilaxis was administered 30 minutes prior to the procedure.

Under sterile conditions, 3 partial-thickness, $5 \mathrm{~cm}$ burns were made on each of the pigs back using a hot plate held in place for 10 seconds (Figure 1). Each of the wounds was immediately covered with either a semiocclusive dressing (Tegaderm $\AA, 3 \mathrm{M}$ ), a cultured keratinocyte patch (Epifast ${ }^{\circledR}$, Bioskinco, Mex.), or stemcell bioscaffold patch (Bioandamio $\AA$ ), Centro Biotecnológico de Terapias Avanzadas, Mex.) (Figure 2). Wounds were appropriately marked. The cultured keratinocyte are human derived, commercially available cryopreserved patches commonly used for burn wounds or skin graft donor sites. The stem-cell bioscaffold is a prefabricated patch containing human derived endometrial stem cells nested on an absorbable collagen scaffold that can be applied directly over the injured skin.

Bioandamio ${ }^{\circledR}$ is manufactured by Alternativas Biomédicas y Regenerativas in Mexico City. The bioscaffold stem-cell patches are manufactured ondemand for each specific case. After evaluation of the donor and screening for particular diseases, mesenchymal stem-cells are isolated from endometrial or placental tissue. Once harvested, cells are expanded in vitro until they reach a number high enough to be able to produce the patches, which usually have a 2-3 million cells $/ \mathrm{kg}$ dose.

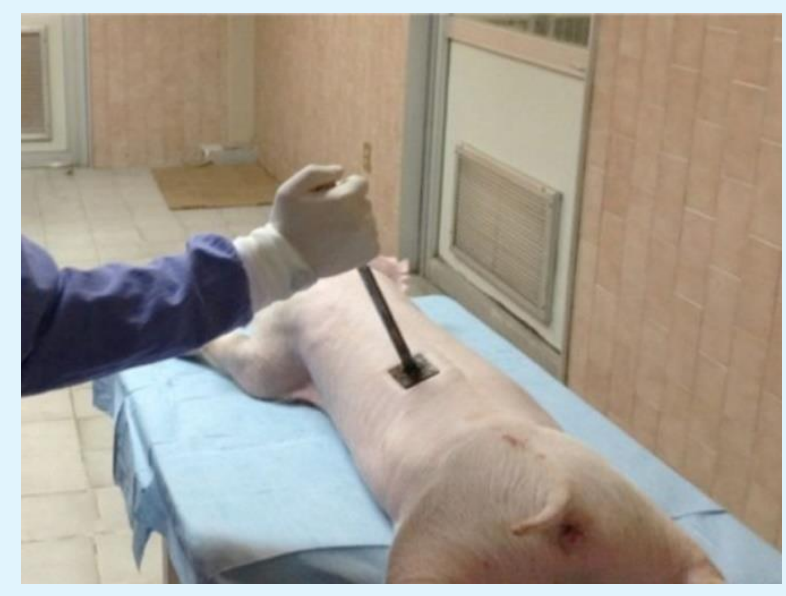

Figure 1: Burn wound with a hot plate on the specimen back.

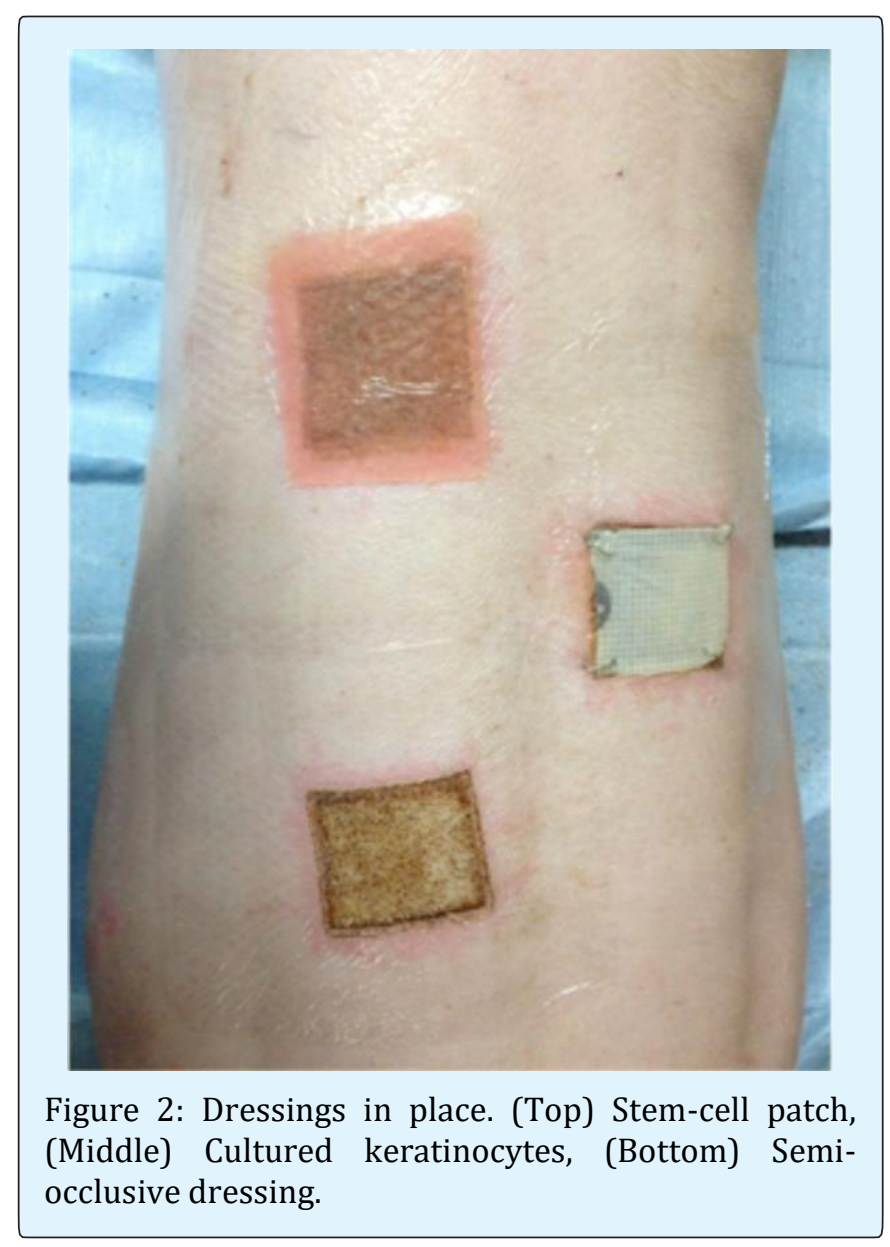

\section{Postoperative care}

Penicillin treatment was continued and metamizole added for the first 2 days. Animals were followed for 15 days. Bandages and bioscaffold patches were changed on day 8 and the new ones kept until day 15; cultured keratinocytes and semi occlusive patches remained until day 15. Pigs were kept on individual cages, at room temperature and fed ad libitum.

\section{Biopsies and histologic study}

On postoperative day 15 , subjects were anesthetized as previously, a $0.5 \mathrm{~cm}$ punch biopsy was taken from each wound and fixed in a $10 \%$ formalin solution. The pathologist was blinded to each of the samples studied. Histologic analysis samples were stained with H-E; the stratum corneum, epidermis and dermis were studied under conventional light microscopy. A grading scale was developed for findings on each of the layers (Table 1). 


\section{International Journal of Transplantation \& Plastic Surgery}

\begin{tabular}{|c|c|c|c|}
\hline \multirow{2}{*}{ Score } & \multicolumn{3}{|c|}{ Findings } \\
\hline & Stratum & Corneum Epidermis & Dermis \\
\hline 1 & Absent & Absent & $\begin{array}{l}\text { Mildly inflamed and } \\
\text { congested }\end{array}$ \\
\hline 2 & Thinned & Dettached & Moderately inflamed and granulated \\
\hline 3 & Inflamed/Thickenned & Eroded & Abundant granulation tissue and vascularization \\
\hline 4 & Normal & Normal & Normal scar \\
\hline
\end{tabular}

*Scores were given according to the closest matching findings.

Table 1: Scoring scale for histologic analysis.

\section{Data Collection and Statistical Analysis}

Data for each group (semi-occlusive, keratinocytes, stem-cell) and variable were recorded on a spreadsheet (Microsoft Excel). Statistical analysis for group comparison was performed with a Mann-Whitney U test. AKruskal-Wallis test with post-hoc Dunn testing was also performed to further validate our findings. Values of $\mathrm{p}<$ 0.05 were considered statistically significant. All statistical analyses were performed using SPSS 19 (IBM).

\section{Results}

We analyzed 10 wounds in each group. Mean histologic scores for each group are shown in Table 2. On statistical analysis, keratinocyte patches showed no significant difference over semi-occlusive patches for any of the skin layers. Stem-cell patches did show a statistically significant difference over semi-occlusive patches on all skin layers studied. When keratinocytes and stem-cell patches were compared, a difference was found only on the dermal layer in favor of the stem-cell patch (Figures $3 \& 4$, Table 3).

\begin{tabular}{|c|c|c|c|}
\hline \multirow{2}{*}{ Skin Layer } & \multicolumn{3}{|c|}{ Group } \\
\cline { 2 - 4 } & Semi-occlusive patch & Culutred Keratinocytes & Stem-cell patch \\
\hline Stratum Corneum & 1.4 & 1.9 & 2.8 \\
\hline Epidermis & 3.1 & 3.5 & 3.7 \\
\hline Dermis & 2 & 1.7 & 2.9 \\
\hline
\end{tabular}

Table 2: Mean histologic scores by skin layer and group.

\begin{tabular}{|c|c|c|}
\hline \multicolumn{3}{|c|}{ Stratum Corneum } \\
\hline Groups & Keratinocyte & p value \\
\hline Semi-occlusive & Stem-cell patch & 0.171 \\
\hline Semi-occlusive & Stem-cell patch \\
\hline Keratinocytes & Epidermis \\
\hline \multicolumn{3}{|c|}{} \\
\hline Groups & Keratinocyte & p value \\
\hline Semi-occlusive & Stem-cell patch & 0.154 \\
\hline Semi-occlusive & Stem-cell patch & $0.032^{*}$ \\
\hline Keratinocytes & Dermis & 0.302 \\
\hline Groups & Keratinocyte & p value \\
\hline Semi-occlusive & Stem-cell patch & 0.504 \\
\hline Semi-occlusive & Stem-cell patch & $0.049^{*}$ \\
\hline Keratinocytes &
\end{tabular}

*Statistically significant difference found.

Table 3: Group comparison by skin layer. 


\section{International Journal of Transplantation \& Plastic Surgery}

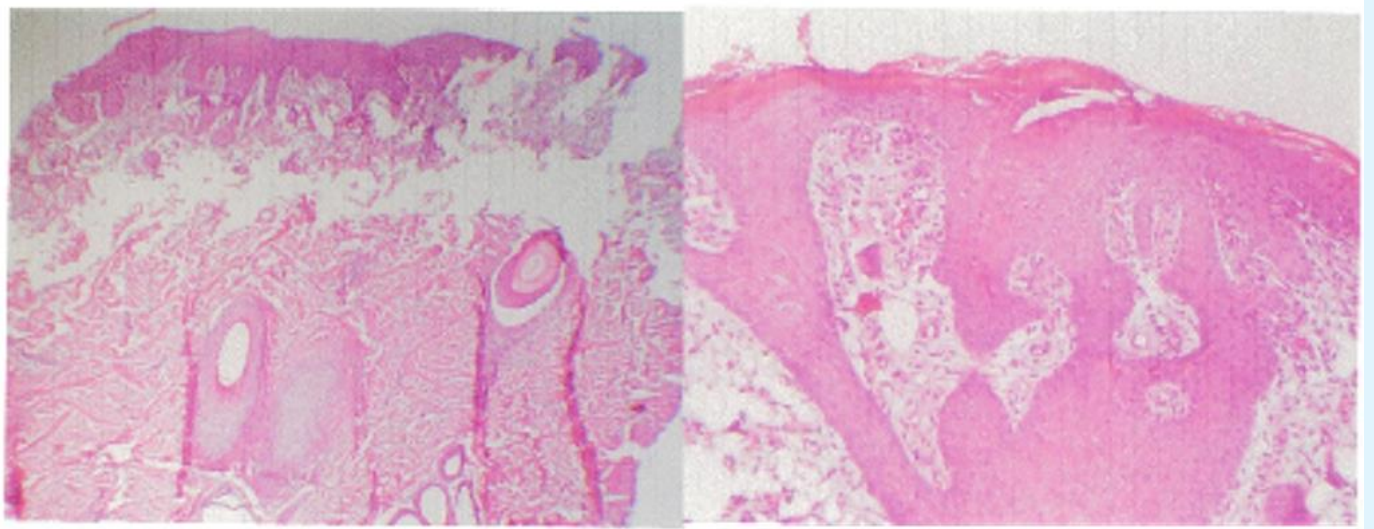

Figure 3: (Left) Cultured Keratinocytes. Note irregular epithelization with epidermis detachment. (Right) Stem-cells. Complete epithelization, epidermal hyperplasia.

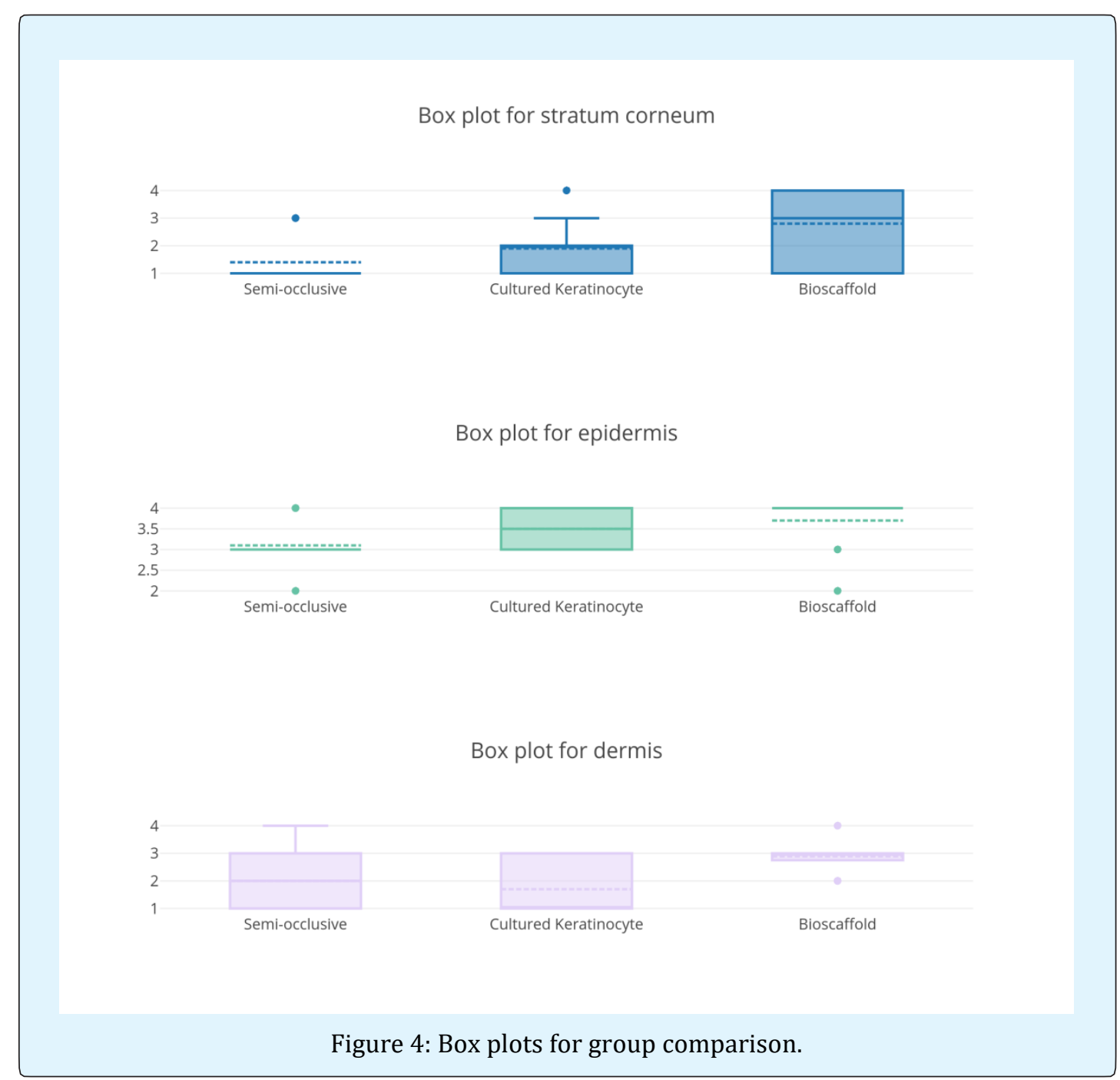




\section{International Journal of Transplantation \& Plastic Surgery}

\section{Discussion}

Wound re-epithelization is a top-most priority in the burnt patient. Over the years, many strategies have been developed in order to hasten the healing process while reducing the consequences of chronic inflammation, such as hypertrophic, painful or retractile scars. Occlusive dressings along with ointments and topical immunomodulators are the mainstay of treatment today. Results are still far from ideal.

Grafting of the wound at a cellular level has been attempted for years. Cultured keratinocytes were postulated as a potential method for faster reepithelization as far back as the 1950's, however, only recently laboratory technology made them commercially available [6]. Cultured keratinocytes are widely used in burn centers around the world $[7,8]$. They have shown to be beneficial in providing early coverage and a functional result. A review of keratinocyte delivery to the wound bed [7], however, they are hard to obtain, difficult to culture, expensive, and need special care for transportation, management and storage [9]. Keratinocyte grafts have a rather low 'take' rate, and proper preparation of the recipient bed is fundamental for their success. They are fully mature differentiated cells that don't modify much of the wound inflammatory environment. Most keratinocyte culture preparations include some epithelial stem cells (EpSC), usually derived from hair follicles or skin glands during harvesting. Preservation of these EpSC is fundamental for a successful and long term survival of the graft, since they possess the greatest proliferative capacity. It has been suggested that loss of a graft after a successful initial take arises from depletion of EpSC [10].

There has been interest in using stem cells for burns and other acute and chronic wounds. Stem cells close wounds faster, prevent scar contracture and potentially restore skin appendages [11-15]. Stem-cells promise to aid in wound healing in 2 ways: stimulating faster cell proliferation and decreasing the inflammatory response. The latter anti-inflammatory effect may even extend to a systemic level resulting in better outcomes and survival for the severely burned.

The therapeutic power of stem-cells resides in their clonogenicity and potency [2]. They are classified according to their potential to differentiate into other cells: totipotent or omnipotent stem-cells can turn into any embryonic or extra embryonic cell; pluripotent stemcells are usually embryonic in origin and can differentiate into any cell of the three germinal layers; multipoint,

Villasenor Villalpando E,et al. Mesenchymal Stem-Cells Patches Improve Burn Wound Reepithelization in a Porcine Wound Model: A Prospective, Comparative Study. Int J Transplant \& Plastic Surg 2018, 2(3): 000125. oligopotent and unipotent stem-cells have fewer differentiation capacity [16].

Allogenic topical multipotent mesenchymal stem-cells (MSC) significantly accelerate wound healing in mice models, achieving higher percentages of rerepithelization, cellularity and angiogenesis $[5,17,18]$.

Mesenchymal stem cells can be harvested from various adult tissues [3] and are relatively easy to expand in vitro, hence they are considered as a potential source of stem cells for building dermal substitutes [4,5,19]. They secrete many growth factors that stimulate wound healing-particularly IGF-1, which has recently shown to be crucial for tissue repair-but produce only low IL-6 levels as compared to fibroblasts. The most common sources of mesenchymal stem-cells are bone marrow and umbilical cord, however each one poses some drawbacks of its own.

Immediately after burn injuries MSCs in the bone marrow multiply and migrate to the injured sites [2], contributing to autolysis of necrotic tissue and neovascularizacion. Bone marrow MSC successfully close non-healing diabetic ulcers when applied topically and injected into the edge of the wound [20-23]. Furthermore, when administered systemically MSC migrate and deposit directly on chronic wounds. The problem with bone marrow stem cells is that they are hard to obtain, usually need to be harvested from the same patient and their availability is scarce. On top of that, there is a well known bone marrow depletion phenomenon in the severely burnt. Because of these disadvantages, other sources of MSC are needed.

Umbilical cord stem-cells are multi potential mesenchymal cells that can differentiate into endothelium, chondrocytes, osteocytes, adipocytes, fibroblasts and myocytes. They have been succesfully used in the burnt patients, but harvesting of umbilical stem cells is difficult, they are expensive to maintain, need to be prepared specifically for the patient and cannot be produced on demand.

Endometrial stem cells (ESC) harvested from menstrual blood have a stable karyotype after laboratory manipulation. They represent and abundant source for mesenchymal stem-cells that raises little ethical issues. ESC have a great angiogenic potential and are especially useful in ischemic tissues [24].

It's clear and widely known that stem-cells contribute to faster and better wound healing, however the optimal 


\section{International Journal of Transplantation \& Plastic Surgery}

source and method for delivery are still to be determined. Manufacturing of a readily available stem-cell derived dermal substitute wound represents a leap forward in the treatment of burnt patients, and even if there is no definitive evidence to favor systemic over local use of stem cells, there are some studies that suggest that acellular matrices or scaffolds increase cell homing, differentiation, mobilization and adhesion [25].

We experimented with ESC nested on a collagen bioscaffold. Our study found that the stem-cell bioscaffold effectively promotes healing and epithelization on acute burn wounds in all three layers studied: corner, epidermis and dermis. The stem-cell bioscaffold is as opposed to cultured keratinocytes composed of bioactive molecules and live troncal endometrial cells which allow for an organized orchestration and effective regenerative process. The particular product we used can be manufactured on demand and be available in only 4 hours, which can prove very useful for treating massive burn casualties. The fact that human stem-cells were viable in a pig wound further demonstrates the immunotolerance mechanisms of such celular grafts.

Additionally, we compared the stem-cell bioscaffold patches to other commonly used topical wound dressings. It proved to be more effective than semi-occlusive dressings for healing in all three layers analyzed. It also proved to be more effective than cultured keratinocytes in the dermal layer. Interestingly, cultured keratinocytes did not show to be more effective than semi-occlusive dressings in any of the layers studied. Clinical findings were in tune with pathological analysis (Figure 5).

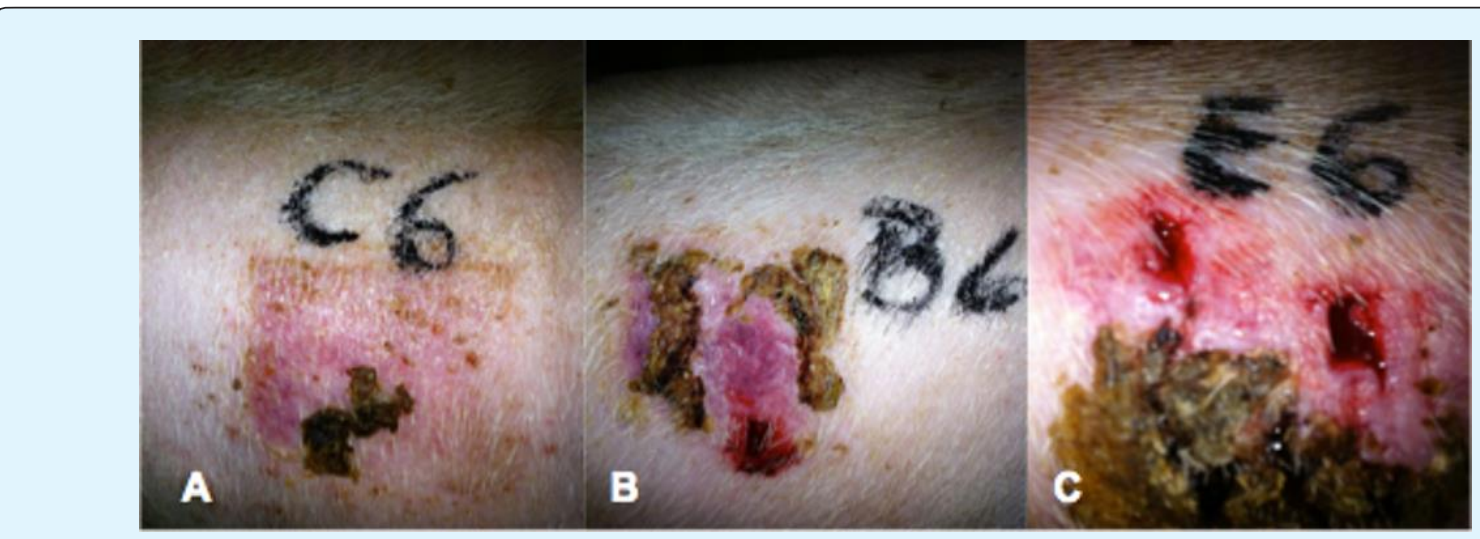

Figure 5: Clinical findings. (A) Stem-cell patch. (B) Semi-occlusive dressing. (C) Cultured Keratinocytes.

An additional difference between the patches used is the presence of collagen in the stem-cell bioscaffold. Collagen was chosen as a substrate for the scaffold because it is a fundamental component of skin that in theory should not elicit inflammatory effects on the surrounding tissues. Although collagen is intended to function only as a carrier for the stem-cells, we did not study if - by itself- collagen has any beneficial effects on wound healing, which could also account for the improved epithelization.

While long-term skin quality is hard to be predicted at 15 days, our goal was to measure time to early epithelization since it represents the restitution of the dermal barrier. At 15 days, the inflammatory phase should be mostly resolved. After that, skin quality can be dependent on many factors such as compression garment therapy or immunomodulating topical agents.

It is worth mentioning that although we tried to objectivize our histologic findings as much as possible, the scoring scale we used was devised post hoc based on the results of histologic results, and the scale we used was not validated, all of which of course is a potential bias for our results. However, we wanted to try and be as objective as possible measuring dermal cells and components, and compare results between the groups. We also want to mention that although we initially intended to determine the presence of dermal appendages, we could not find them in any of the samples studied; perhaps there was not enough time for them to develop. 


\section{International Journal of Transplantation \& Plastic Surgery}

\section{Conclusions}

Endometrium derived mesenchymal stem-cells are an abundant and easy to harvest source for prefabrication of live cellular dressings. They are effective in attaining a faster and more organized wound healing, even in crossspecies grafting.

\section{References}

1. Pittenger MF, Mackay AM, Beck SC, Jaiswal RK, Douglas R, et al. (1999) Multilineage potential of adult human mesenchymal stem cells. Science 284(5411): 143-147.

2. Butler KL, Goverman J, Ma H, Fischman A, Yu YM, et al. (2010) Stem cells and burns: review and therapeutic implications. J Burn Care Res 31(6): 874881.

3. Dieckmann C, Renner R, Milkova L, Simon JC (2010) Regenerative medicine in dermatology: biomaterials, tissue engineering, stem cells, gene transfer and beyond. Exp Dermatol 19(8): 697-706.

4. Fukumoto T, Sperling JW, Sanyal A, Fitzsimmons JS, Reinholz GG, et al. (2003) Combined effects of insulinlike growth factor-1 and transforming growth factorbeta1 on periosteal mesenchymal cells during chondrogenesis in vitro. Osteoarthritis Cartilage 11(1): 55-64.

5. Le Blanc K, Tammik C, Rosendahl K, Zetterberg E, Ringdén $O$ (2003) HLA expression and immunologic properties of differentiated and undifferentiated mesenchymal stem cells. Experimental Hematology 31(10): 890-896.

6. Langdon RC (1989) Using Cultured Keratinocytes for Treating Burns. West J Med 151(6): 655-656.

7. Wood FM, Kolybaba ML, Allen P (2006) The use of cultured epithelial autograft in the treatment of major burn injuries: A critical review of the literature. Burns 32(4): 395-401.

8. Chester DL, Balderson DS, Papini RPG (2004) A review of keratinocyte delivery to the wound bed. J Burn Care Rehabil 25(3): 266-275.

9. Atiyeh BS, Costagliola M (2007) Cultured epithelial autograft (CEA) in burn treatment: Three decades later. Burns 33(4): 405-413.
10. Pellegrini G, Ranno R, Stracuzzi G, Bondanza S, Guerra L, et al. (1999) The control of epidermal stem cells (holoclones) in the treatment of massive fullthickness burns with autologous keratinocytes cultured on fibrin. Transplantation 68(6): 868-879.

11. Martin I, Muraglia A, Campanile G, Cancedda R, Quarto R (1997) Fibroblast growth factor-2 supports ex vivo expansion and maintenance of osteogenic precursors from human bone marrow. Endocrinology 138(10): 4456-4462.

12. Zou Z, Zhang Y, Hao L, Wang F, Liu D, et al. (2010) More insight into mesenchymal stem cells and their effects inside the body. Expert Opin Biol Ther 10(2): 215-230.

13. Sheng Z, Fu X, Cai S, Lei Y, Sun T, et al. (2009) Regeneration of functional sweat gland-like structures by transplanted differentiated bone marrow mesenchymal stem cells. Wound Repair Regen 17(3): 427-435.

14. Li H, Fu X, Ouyang Y, Cai C, Wang J, et al. (2006) Adult bone-marrow-derived mesenchymal stem cells contribute to wound healing of skin appendages. Cell Tissue Res 326(3): 725-736.

15. Huang S, Lu G, Wu Y, Jirigala E, Xu Y, et al. (2012) Mesenchymal stem cells delivered in a microspherebased engineered skin contribute to cutaneous wound healing and sweat gland repair. J Dermatol Sci 66(1): 29-36.

16. Schöler HR (2007) The Potential of Stem Cells: An Inventory. In: Nikolaus K, Dagmar S, Sorgner SL, (Eds.), Human biotechnology as Social Challenge. Ashgate Publishing, pp: 28.

17. Foresta C, Schipilliti M, De Toni L, Magagna S, Lancerotto L, et al. (2011) Blood levels, apoptosis, and homing of the endothelial progenitor cells after skin burns and escharectomy. J Trauma 70(2): 459-465.

18. Cheifetz S, Bellón T, Calés C, Vera S, Bernabeu C, et al. (1992) Endoglin is a component of the transforming growth factor-beta receptor system in human endothelial cells. J Biol Chem 267(27): 19027-19030.

19. Shake JG, Gruber PJ, Baumgartner WA, Senechal G, Meyers J, et al. (2002) Mesenchymal stem cell implantation in a swine myocardial infarct model: 


\section{International Journal of Transplantation \& Plastic Surgery}

engraftment and functional effects. Ann Thorac Surg 73(6): 1919-1926.

20. Rasulov MF, Vasilchenkov AV, Onishchenko NA, Krasheninnikov ME, Kravchenko VI, et al. (2005) First experience of the use bone marrow mesenchymal stem cells for the treatment of a patient with deep skin burns. Bull Exp Biol Med 139(1): 141-144.

21. Vojtassák J, Danisovic L, Kubes M, Bakos D, Jarábek L, et al. (2006) Autologous biograft and mesenchymal stem cells in treatment of the diabetic foot. Neuro Endocrinol Lett 27(2): 134-137.

22. Falanga V, Iwamoto S, Chartier M, Yufit T, Butmarc J, et al. (2007) Autologous Bone Marrow-Derived Cultured Mesenchymal Stem Cells Delivered in a Fibrin Spray Accelerate Healing in Murine and Human Cutaneous Wounds. Tissue Eng 13(6): 1299-1312.
23. Ravari H, Hamidi-Almadari D, Salimifar M, Bonakdaran S, Parizadeh MR, et al. (2011) Treatment of non-healing wounds with autologous bone marrow cells, platelets, fibrin glue and collagen matrix. Cytotherapy 13(6): 705-711.

24. Murphy MP, Wang H, Patel AN, Kambhampati S, Angle $\mathrm{N}$, et al. (2008) Allogeneic endometrial regenerative cells: an "Off the shelf solution" for critical limb ischemia? J Transl Med 6: 45.

25. Ichioka S, Kouraba S, Sekiya N, Ohura N, Nakatsuka T (2005) Bone marrow-impregnated collagen matrix for wound healing: Experimental evaluation in a microcirculatory model of angiogenesis, and clinical experience. Br J Plast Surg 58(8): 1124-1130. 